\title{
Programa Mais Médicos:
percepção dos usuários
e dos profissionais do SUS
}

Brazil's More Doctors Program:

Users and Health Professionals' Perceptions

\author{
Josiane Fernandes Lozigia Carrapato* \\ Ricardo Verpa Costa da Silva** \\ Eliza Carloni Rotondaro*** \\ Nádia Placideli****
}

\begin{abstract}
Resumo - Este trabalho tem por finalidade demonstrar o desempenho dos médicos do Programa Mais Médicos em município de pequeno porte do interior do estado de São Paulo, bem como evidenciar a percepção dos usuários e profissionais da Estratégia Saúde da Família. Para tanto, realizou-se busca na literatura nacional, abordando assuntos referentes ao Sistema Único de Saúde, à Saúde da Família e ao Programa Mais Médicos. Trata-se de um estudo qualitativo, por meio da técnica de entrevista com profissionais das unidades de saúde e com os usuários assistidos pelos médicos do programa. Os resultados demonstraram que o atendimento médico dos profissionais vinculados ao Programa Mais Médicos ocorre de maneira qualificada, humanizada e resolutiva, trazendo, assim, atendimento de qualidade aos usuários dos serviços de saúde.

Palavras-chave: estratégia de saúde da família; Sistema Único de Saúde; atenção primária de saúde.

Abstract - This paper aims to show the performance of physicians of the More Doctors program in small towns in the state of São Paulo, as well as the perception of the public and of Family Health Strategy's employees. The research encompassed the national literature in the
\end{abstract}

\footnotetext{
* Doutoranda em Saúde Coletiva pela Faculdade de Medicina da UNESP de Botucatu. Docente da graduação do Centro Universitário de Bauru - ITE para o curso de Serviço Social. Docente do curso de pós-graduação da Universidade Sagrado Coração - USC, do Instituto Passo 1 e do Centro Universitário de Bauru - ITE. Assistente Social da Secretaria Municipal de Saúde. Correspondência: Rua Matilde Fraga Moreira de Almeida, n. 7/70 - Parque São João, Bauru -São Paulo. CEP: 17055-240. Email: <jo.carrapato@uol.com.br>.

** Pós-graduação em Administração em Saúde; Aprimoramento de qualificação de UBS e em Gestão de Políticas Públicas, Organizacional e Terceiro Setor. Secretário Municipal de Saúde na Secretaria Municipal de Saúde de Macatuba. Correspondência: Rua Coronel Virgílio Rocha, 22-60 - Centro. Macatuba - SP. CEP 17290-000. Email: <ricardo verpa@hotmail.com>.

*** Pós-graduação em Gestão de Políticas Públicas, Organizacional e Terceiro Setor. Assistente Social na Santa Casa de Misericórdia de Duartina - Hospital Santa Luzia. Correspondência: Rua Dona Rima Simão nº 638 - Centro, Duartina - São Paulo.CEP: 17.470-000. Email:<eliza_carloni_22@hotmail.com>.

**** Doutoranda em Saúde Pública pela Faculdade de Medicina de Botucatu (FMB-UNESP). Bolsista de doutorado pela CAPES. Correspondência: Rua Santa Catarina n. 126 - Cruzeiro, Lençóis Paulista - São Paulo. CEP: 18.680-500. Email:<nadiaplacideli@hotmail.com>.
} 
field, covering matters related to the National Health System, Family Health, and the More Doctors program. It is a qualitative study, using interviews with health units' employees and users assisted by the program to demonstrate that the medical care provided by doctors associated with the More Doctors program is of quality, humanized, and resolutive, thus bringing quality care to users of health services. Keywords: Family Heath Strategy; Unified Health System; Primary Health Care.

\section{Introdução}

A Constituição Federal de 1988 foi um passo importante para a democratização do acesso igualitário e universal à saúde, com a criação de um novo modelo de assistência. As mudanças destacadas contidas na nova legislação são fruto de reivindicação social e política da sociedade brasileira.

O Sistema Único de Saúde (SUS), garantido pela Constituição Federal de 1988 e regulamentado pelas Leis Orgânicas de Saúde n. 8.080 e n. 8.142 de 1990, constitui grande avanço na consolidação da Reforma Sanitária Brasileira (BRASIL, 1988). Entretanto, ainda enfrenta o desafio de implementar um modelo de atenção integral à saúde dos indivíduos, famílias e comunidade. Esse modelo pós-reforma, representado pelo SUS, consolida conceitos e práticas como acolhimento, humanização, acesso universal, integridade da atenção e vínculo com o objetivo de resgatar a relação entre os sujeitos sociais (VASCONCELOS; GRILLO; SOARES, 2009).

O SUS consolidou-se, ao longo de duas décadas, como a maior política de Estado do país, promotor de inclusão e justiça social. Fruto de uma permanente construção coletiva, nele se manifesta o melhor da tradição política brasileira: o diálogo, a composição e a busca pelo acordo. Dessa maneira, é um dos maiores exemplos de política pública no Brasil, mesmo com toda dificuldade encontrada atualmente no que se refere às questões do financiamento do Sistema Público de Saúde. Os governos têm como obrigação a garantia da universalidade, equidade e integralidade, buscando a plenitude da saúde da população dividida nas esferas federal, estadual e municipal.

O Ministério da Saúde criou o Programa de Saúde da Família (PSF), atualmente denominado Estratégia Saúde da Família (ESF), na tentativa de superar o modelo hegemônico centrado em práticas curativas e na atenção médico-hospitalar presente nos municípios. Este programa surgiu no ano de 1994 como uma estratégia de organização das ações de saúde nos serviços da atenção básica, fundamentado em um novo modelo de atenção, com ênfase na promoção da saúde dos indivíduos, famílias e da comunidade. Sua implantação possibilitou ampliar o acesso da população aos serviços 


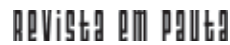

\} PROGRAMA MAIS MÉDICOS - CARRAPATO, J. F. L.; SILVA, R. V. C.; OTONDARO, E. C.; PLACIDELI, N. \}

DOI: $10.12957 /$ REP.2016.27863

de saúde, desenvolver práticas de saúde integrada com as famílias, incorporar o atendimento humanizado e fomentar o compromisso com as necessidades dos usuários (SOUSA, 2008).

Apesar de todos os benefícios alcançados durante as implementações das equipes de Estratégia Saúde da Família para o atendimento aos usuários, é possível notar o aumento da demanda espontânea e a sobrecarga de trabalho dos profissionais. O excesso de demanda por consultas médicas nas unidades de saúde limita o desenvolvimento de ações programáticas e a ênfase na promoção da saúde e prevenção de doenças.

O Programa Mais Médicos, por sua vez, faz parte de um amplo pacto de melhoria do atendimento aos usuários do SUS, que prevê mais investimentos em infraestrutura dos hospitais e unidades de saúde, além de levar mais médicos para regiões onde há escassez e ausência de profissionais (CARRYL; ROSSATO; PRADOS, 2016). Segundo Campos (2015), a contratação de médicos para a rede de atenção básica (primária) em área de grande vulnerabilidade social e sanitária é importante, mas não solucionará o problema de saúde pública no Brasil.

O Brasil contratou profissionais médicos de governos parceiros do programa para atender e suprir a falta destes profissionais no país, principalmente em áreas de maior vulnerabilidade, com a criação do Programa Mais Médico, desde o ano de 2013 (CARRYL; ROSSATO; PRADOS, 2016).

É importante pensar a saúde para além de contratação de médicos e, além disso, é fundamental romper com o modelo tradicional de serviço de saúde centrado na doença. Temos que considerar os determinantes sociais e econômicos presentes na população que constituem realmente a causa das doenças. Tendo isso em vista, muitas doenças poderiam ser prevenidas com a efetivação de estratégias direcionadas aos indivíduos de alto risco e aos indivíduos ainda saudáveis. Estes, se não receberem informações acerca dos malefícios do comportamento alimentar inadequado, da bebida alcoólica, do tabagismo e da falta de atividades físicas poderão acabar se tornando pessoas "doentes" (CAMPOS, 2015).

Ribeiro (2015) refere que há alguns equívocos relacionados ao Programa Mais Médicos, sendo o primeiro deles a definição do problema centrado no corpo, na doença, sem qualquer tentativa de reflexão sobre outras causas. O segundo são as propostas equivocadas de enfrentamento do problema, as quais, com uma visão dominada por uma perspectiva médica e com foco na doença, reduzem as ações através de medicamentos e cirurgias. Nessa lógica é necessário que o indivíduo adoeça para então tratar a sua doença. O terceiro equívoco são propostas desconectadas das evidências científicas relevantes.

A OMS elaborou em 2003 uma diretriz denominada Estratégia global sobre dieta, atividade física e saúde, lembrando o quanto é importante iniciar hábitos saudáveis nas crianças e modificar os hábitos dos adultos. Desde o ano de 2000 há a Estratégia global para prevenção e controle das 


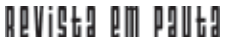

\} PROGRAMA MAIS MÉDICOS - CARRAPATO, J. F. L.; SILVA, R. V. C.; OTONDARO, E. C.; PLACIDELI, N. \}

DOI: $10.12957 /$ REP.2016.27863

doenças crônicas não transmissíveis, buscando despertar, junto aos profissionais, novas formas de se fazer saúde.

Com o propósito de garantir, ampliar e melhorar o acesso a toda a população, o Brasil lança o Programa Mais Médicos, em que profissionais médicos prestam serviços em milhares de municípios do país, levando até a comunidade o direito do atendimento em saúde, a busca por tratamento de doenças, o controle de patologias existentes e nunca antes avaliadas, os conhecimentos sobre uso de medicação e a educação em saúde. O foco principal são indivíduos que nunca tiveram atendimento médico durante sua vida e que através do programa têm o primeiro acesso, a primeira consulta, a primeira orientação, possibilitando a melhora da qualidade de vida aos brasileiros (BRASIL, 2013).

Por meio da Lei n. 12.871 de 22 de outubro de 2013 (BRASIL, 2013), com a convocação de médicos para atuar em serviços de atenção primária, nas periferias de grandes cidades e municípios do interior do país, o governo federal garantirá mais médicos para o Brasil e mais saúde à população.

Este trabalho tem como objetivo evidenciar a percepção dos profissionais e da população usuária do SUS sobre o Programa Mais Médicos, bem como traçar o relacionamento entre os médicos do programa, os profissionais da equipe de saúde e os usuários.

\section{Metodologia da pesquisa e análise dos resultados}

Trata-se de uma pesquisa qualitativa, na qual inicialmente procuramos explorar o assunto sobre o Programa Mais Médicos e, posteriormente, descrever os resultados encontrados.

Na coleta de dados, utilizamos a técnica de entrevista, sendo a mesma essencial para conhecermos a subjetividade dos sujeitos. A entrevista é um instrumento facilitador para a pesquisa, pois os sujeitos têm oportunidade de expressarem livremente o pensamento através da linguagem verbal.

A entrevista estruturada desenvolve-se a partir de uma relação fixa de perguntas, cuja ordem e redação permanece invariável para todos os entrevistados, que geralmente são em grande número. Por possibilitar o tratamento quantitativo dos dados, este tipo de entrevista torna-se o mais adequado para o desenvolvimento de levantamentos sociais. (GIL, 2008, p. 113).

Dessa maneira, realizamos entrevista com dez profissionais de unidade de Saúde da Família, incluindo enfermeiros, técnicos de enfermagem, agentes comunitários de saúde, agentes de controle de vetores, assessores técnicos e agentes de transporte. Também realizamos entrevista 


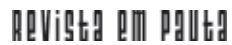

\} PROGRAMA MAIS MÉDICOS - CARRAPATO, J. F. L.; SILVA, R. V. C.; OTONDARO, E. C.; PLACIDELI, N. \}

DOI: $10.12957 /$ REP.2016.27863

com dez usuários. Ou seja, a amostra foi intencional e composta por 20 sujeitos, sendo dez profissionais e dez usuários.

Após levantamento da coleta de dados, os mesmos passaram por um processo de análise, através do método de análise de conteúdo.

A análise de conteúdo é um tema central para todas as ciências humanas e com o transcurso do tempo tem-se transformado em um instrumento importante para o estudo da interação entre os indivíduos. Ou seja, o estudo dos símbolos e das características da comunicação é básico para compreender o homem, sua história, seu pensamento, sua arte e suas instituições. (RICHARDSON, 1985, p. 175).

Assim, a estrutura do texto está organizada a partir das categorias emergentes da realidade estudada: percepção dos profissionais do SUS sobre o Programa Mais Médicos e percepção dos usuários do SUS sobre o mesmo programa.

A pesquisa foi realizada em um município de pequeno porte do interior do estado de São Paulo, bem como submetida e aprovada pela comissão de ética e pesquisa da coordenação de saúde desse município. A população é de aproximadamente 19.400 habitantes, de acordo com dados do IBGE (2010), e recebeu profissionais médicos naturais de Cuba, como ocorreu em muitos outros municípios paulistas.

A fim de manter o anonimato dos sujeitos da pesquisa, para descrição dos resultados foram utilizados números de um a dez na referência aos profissionais e usuários (profissionais: 1, 2, 3, 4, 5, 6, 7, 8, 9, 10; usuários: $1,2,3,4,5,6,7,8,9,10)$.

\section{Análise dos resultados}

O presente estudo foi realizado com os profissionais e com os usuários de serviços ESF com Programa Mais Médicos a fim de caracterizar a percepção e o relacionamento dos profissionais e usuários do SUS com os profissionais deste programa.

Nota-se que, antes, muitos dos usuários pesquisados encontravam dificuldade para se expressarem nas consultas médicas de rotina, pois havia um grande fluxo de pacientes para serem atendidos pelo médico da família, tornando assim a consulta muito rápida.

Constata-se que os pacientes se mostraram satisfeitos com as alterações e mudanças que vêm ocorrendo na saúde pública e no SUS, pois agora é possível receber atendimento médico com maior tempo, em consultas nas quais ocorrem explicações sobre os pedidos de exames e sobre as medicações prescritas. Desta forma, os profissionais reconheceram que é necessário buscar técnicas de atendimento humanizado, participativo, explicativo e ético, proporcionando bem-estar aos usuários. 
Observa-se, através dos relatos dos profissionais pesquisados, que o Programa Mais Médicos foi um acréscimo satisfatório à população, proporcionando um atendimento médico mais elaborado e participativo. Já com os usuários pesquisados ficou nítida a aceitação, a confiança e a admiração que eles sentem pelos médicos do programa, sendo relatados ainda muitos elogios referentes à conduta do atendimento médico prestado por eles.

A prática profissional se torna cada dia mais complexa devido às expressões das questões sociais presentes na sociedade atual. Com isso, é necessário que ocorra a continuidade e a longitudinalidade do cuidado na atenção primária (STARFIELD, 2002), visando melhoria na qualidade de vida do paciente.

A prática profissional da equipe dos funcionários da ESF visa efetivar e emancipar os usuários nas práticas de saúde, com atendimento adequado, mediante ações desenvolvidas em equipe.

\section{Percepção dos profissionais do SUS sobre o Programa Mais Médicos}

Ao serem questionados acerca do que os profissionais imaginavam sobre o médico do Programa Mais Médicos, os sujeitos pesquisados relataram que o médico do programa auxiliaria na saúde pública, especificamente no déficit de médicos existente no Brasil.

Imaginei que ia trazer mais médicos para o país e que supriria a falta dos médicos do posto de saúde e na cidade em geral. (Profissional 1).

Imaginava que seria bem diferente do que está sendo. Achei que eles não iriam atender bem os pacientes. (Profissional 10).

Eu achava que o atendimento seria diferente e não igual está sendo, que é um atendimento bem melhor que os brasileiros, pois eles têm um contato muito maior com os pacientes. Imaginava que seria mais ou menos igual ao que é médico da família. Só que eles não, eles são mais atenciosos com os pacientes, visitam a casa, escutam, enfim, cuidam das pessoas. (Profissional 2).

Que era um programa bom que veio para ajudar, pois faltava médico. Veio pra todo mundo ser atendido, ampliar o número de pessoas atendidas. (Profissional 3).

Imaginava que viria profissional para atender pacientes nas áreas mais carentes e mais afastadas da cidade. (Profissional 5).

Achava que seria bom. Mas, imaginava que não iríamos conseguir entender o que ela fosse falar, por ser diferente o vocabulário e o idioma. (Profissional 8). 
Imaginava que seria bem diferente do que está sendo. Achei que eles não iriam atender bem os pacientes, no entanto, eles atendem melhor que os médicos brasileiros. (Profissional 10).

Os profissionais entrevistados, a princípio, estavam preocupados sobre como seria o processo de adaptação desses médicos na realidade brasileira, no entanto, foram surpreendidos com a qualidade do atendimento prestado principalmente na área de escuta ampliada e qualificada. Além disso, eles tinham receio de não entender o idioma falado, mas houve rápida adaptação da fala na realidade de trabalho. Muitos profissionais afirmaram que inicialmente o entendimento da linguagem falada pelos médicos do programa, ou seja, o espanhol, foi um pouco complicado, pois havia palavras de difícil compreensão.

Constatamos, também, que os médicos do Programa Mais Médicos solicitam exames e prescrevem medicamentos apenas quando necessário e procuram conhecer a realidade vivida pelo paciente com visitas domiciliares. Ao questionarmos os profissionais acerca da capacidade de dialogar e compreender as orientações e condutas médicas, estes relataram que até o momento estão conseguindo trabalhar adequadamente com esses profissionais.

É! Às vezes a gente não entende muito, mas elas repetem e falam mais calmas, aí entendemos sim, elas passam bastantes ensinamentos de lá também. (Profissional 2).

Fácil, dá pra entender sim as coisas que ela fala. Ela é bem prestativa, explica, ela faz de tudo para o paciente entender o que ela está falando e explica todo o procedimento certinho. (Profissional 3).

Sim, depois que vieram para a nossa unidade, conseguimos entender e ela também entender a gente, agora está melhorando cada dia mais. (Profissional 5).

Sim, mais às vezes tenho um pouco de dificuldade, então peço para repetir e fala um pouco mais calma, aí consigo sim. (Profissional 6).

Fácil. É um diálogo gostoso, cheio de atenção, de abertura. Ela quer saber tudo do paciente, se toma alguma medicação ou se tem alguma doença. (Profissional 10).

Ao questionarmos os profissionais sobre o conhecimento da política de saúde brasileira, o SUS, foi observado que todos conhecem algo sobre ele. Algumas pessoas afirmaram o conhecimento sobre o SUS, sobre consultas e exames, bem como o fato de que ele se destina principalmente aos indivíduos com menor renda. 
Que devemos atender o paciente como ele chega à unidade, cada um é individual, e todos merecem atendimento com qualidade. (Profissional 5).

Sim! Que tem que atender a população, qualquer pessoa. Que tem que fornecer médico e o atendimento básico e fornecer tudo o que a paciente precisa. Pedido de exame, passar medicação e depois se caso o posto não suprir, mandar para o hospital. (Profissional 1).

Sim! Que tem que ter o direito pra todos e a gente tem que distinguir as pessoas que tão mais necessitadas e dar uma atendimento mais especializados. Tem integralidade e tudo. (Profissional 2).

Sim, prevenir, cuidar, proteger, tratar, recuperar, promover, enfim, produzir e garantir saúde aos usuários do SUS. (Profissional 7).

Sim, é que todos do território brasileiro tenham o direito a atendimento, valorizando o paciente, pedindo exames e proporcionando para a população mais pobre atendimento bom de saúde. (Profissional 10).

Os médicos cubanos estão procurando interagir com a Política Nacional de Saúde e, inclusive, verificamos que muitos estão dando continuidade à maneira com a qual os médicos brasileiros já atendiam, com muitas solicitações de exames e prescrições de medicamentos para os pacientes sentirem-se satisfeitos. No entanto, alguns profissionais entrevistados percebem a diferença do atendimento dos médicos cubanos e elogiam a maneira do atendimento dispensado aos pacientes.

Sim! Elas estudam bastante o sistema aqui do Brasil e estão se adaptando muito bem. (Profissional 1).

Sim, dá atenção e os direitos são pra todos, e procura dar mais atenção para o paciente, acompanha quando é internado e dá atenção ao paciente, se precisar ir na casa mesmo sem ser acamado ele vai e é essa a diferença dos médicos do Brasil. (Profissional 2).

Sim, eles prescrevem o que temos e solicitam o exame de acordo com nossa disponibilidade, estamos sempre em diálogo. (Profissional 5).

Sim, até mesmo nos exames e remédios, atendimentos residenciais eles fazem e muito bem. (Profissional 6).

Agora sim, eles estão se interagindo muito bem. Já estão entendendo mais sobre as nossas medicações e sobre o que os pacientes falam. A saúde tá ótima, diminuiu muito a fila de pacientes com as médicas cubanas. (Profissional 9).

Sim, pois ela perguntou tudo como funcionava antes de começar atender os pacientes. Ela procurou saber qual a função de cada fun- 
cionário, ela vai nas visitas domiciliares e busca saber dos pacientes. (Profissional 10).

Todos os profissionais entrevistados relataram que o atendimento realizado pelos médicos do Programa mais Médicos aos pacientes é de excelente qualidade. Muitas falas afirmam essa qualidade na atenção dispensada aos pacientes com atendimento humanizado, cuidado ampliado, escuta ativa, entre outros.

A inserção dos médicos cubanos no Brasil é vista por todos os profissionais pesquisados como uma possibilidade de melhorar a saúde pública, com preocupação em resolver as dificuldades dos pacientes, ampliar a oferta de atendimento e poder entender a realidade vivida por essas pessoas denominadas "pacientes".

Acho que boa, por que, como eu disse, eles estão conseguindo suprir as necessidades. São bem atenciosos, pedem bastantes exames e todos os pacientes saem satisfeitos do consultório. (Profissional 1).

A qualidade do atendimento é muito boa. Ela não atende o paciente só com uma dor aparente do paciente. Elas pedem exames para verem o paciente como um todo. (Profissional 2).

Atende sim, porque ela é muito atenciosa e dedicada. (Profissional 3).

Ótima. Atende bem, atencioso, compreende o que você tem. É super dez. (Profissional 4).

Sim, com consultas, atenção, coloca a mão no paciente, muito atenciosos. (Profissional 5).

Sim, com muita atenção e muitos conhecimentos, conseguem descobrir o que o paciente tem e tratar de forma correta. (Profissional 6).

Sim, todos os procedimentos com total responsabilidade, segurança e dedicação. (Profissional 7).

Acho. A médica se preocupa muito com os pacientes, não tem pressa para terminar o atendimento com o paciente, faz muitos pedidos de exames e visitas nas casas. (Profissional 8).

Atende. Eles perguntam os sintomas, se preocupam e não têm pressa de terminar o atendimento. (Profissional 9).

Muito, pois eles dão muita atenção ao paciente, pergunta muito e se interessa em saber o que acontece com o paciente. (Profissional 10).

Os profissionais inseridos no SUS acreditam que o Programa Mais Médicos auxiliou muito no atendimento da saúde pública. No que se refere 


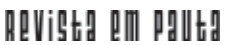

\} PROGRAMA MAIS MÉDICOS - CARRAPATO, J. F. L.; SILVA, R. V. C.; OTONDARO, E. C.; PLACIDELI, N. \}

DOI: $10.12957 /$ REP.2016.27863

a esse trabalho, cabe ressaltar que o município pesquisado situa-se no Centro-Oeste do estado de São Paulo e, anteriormente a esse programa, havia muitas dificuldades na contratação e manutenção dos médicos nas unidades de saúde.

Na percepção dos profissionais, há espaço tanto para os médicos brasileiros quanto para os cubanos, de modo que não é preciso disputa entre os profissionais, pois há necessidade de ampliar a Estratégia de Saúde da Família em todo território brasileiro. Essa ampliação se faz necessária com a contratação de médicos, bem como de outros profissionais, como psicólogos, assistentes sociais, enfermeiros, equipe de nível médio/técnico e equipe de apoio.

\section{Percepção dos usuários do SUS sobre o Programa Mais Médicos}

Ao serem questionados sobre o Programa Mais Médicos, os usuários do SUS são unânimes em afirmar a qualidade dos atendimentos dos médicos cubanos. Verificamos que as pessoas precisam de atenção, pois o que mais impressiona os usuários do SUS é a escuta qualificada, o tempo dispensado durante a consulta, o exame físico e as orientações básicas transmitidas durante o atendimento.

Eu imaginava que a gente não ia entender o que eles iam falar e que eles não iam tratar a gente bem. Tinha medo. Mas depois da primeira vez que passei com ela eu adorei. Ela só passa o remédio depois dos exames. (Usuário 1).

Esperava que as consultas fossem iguais a que está sendo realizada, com atenção e qualidade. (Usuário 3).

Eu achava que era tudo a mesma coisa. Mas é diferente, viu. Ela tem mais paciência, vai mais a fundo, eu achava que era mais ou menos, igual aos outros, que não ia perguntar nada. (Usuário 4).

Imaginava que fossem médicos como os nossos, que iria atender e nem olhar para a nossa cara. (Usuário 7).

Antes de começar eu não tinha noção nenhuma de como seria, ficava até inseguro. Agora que fui atendido com atenção, não quero outra coisa. (Usuário 8).

Os pacientes que são atendidos pelos médicos cubanos afirmaram que o diálogo com o médico é de fácil compreensão. Apenas três usuários relataram dificuldade inicial de manter um diálogo, sendo que após um período ocorreu adaptação e a possibilidade de entendimento das orientações durante a consulta. Já com relação à conduta médica na solicitação de exames e de medicamentos, todos os sujeitos pesquisados relacionam a 


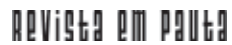

\} PROGRAMA MAIS MÉDICOS - CARRAPATO, J. F. L.; SILVA, R. V. C.; OTONDARO, E. C.; PLACIDELI, N. \}

DOI: $10.12957 /$ REP.2016.27863

qualidade com exames e medicamentos, como se isso fosse essencial na saúde pública.

Conforme estudo realizado por Onocko-Campos et al. (2012), a fim de avaliar estratégias inovadoras para a atenção primária no município de Campinas, em entrevistas realizadas junto aos usuários de unidades ESF, estes afirmaram a importância da implantação de estratégias que qualifiquem a assistência, como conhecer os profissionais, a crítica à rotatividade dos médicos e a inconformidade com as filas.

Além disso, muitos usuários não conhecem o significado do SUS na saúde brasileira; outros, por sua vez, mobilizados e participativos, afirmam conhecer um pouco sobre o SUS.

SUS! Isso daí eu não entendo e não sei não. (Usuário 1).

Não. Eu não conheço e não entendo muito isso. (Usuário 2).

Sim, todos devem ser atendidos de forma igual, sem privilégios, todos com os mesmo direito. (Usuário 3).

O SUS era pra todos, mas os serviços demoram e os médicos não pedem nada, não fazem nada, precisava melhorar um pouco, pois demora muito. (Usuário 5).

Sim, temos direito à saúde e temos direito de cobrar isso do poder público. (Usuário 6).

Ao questionar os usuários do SUS sobre o que eles pensavam acerca do Programa Mais Médicos, todos visualizaram aspectos positivos na possibilidade de serem atendidos pelos médicos cubanos. Isto porque, sem a inclusão desses profissionais no país, seria difícil arrumar tantos médicos para solucionar as dificuldades locais.

Foi bom. Porque os médicos dos postos não estavam dando conta. Agora tem mais sossego pros outros. E às vezes é bom trocar de médico. (Usuário 1).

Acho boa. Porque quando o médico atende a gente bem, tem interesse em descobrir o que a gente tem. (Usuário 2).

Uma boa, pois estava tendo muita reclamação da saúde. Na TV você só vê o povo reclamando. Acho que eles vão endireitar um pouco. (Usuário 4).

Ótimo, estávamos sem médicos aqui, agora temos médico quando estamos passando mal. (Usuário 5).

Acredito que foi uma decisão corajosa do governo federal, acredito também que, para obter o sucesso, os médicos deverão ter condições 
de trabalho, acesso a exames e medicamento, e também transporte. Sou defensora também de valorizar o que temos no nosso país, como médicos, remédios e exames. (Usuário 7).

Que estão momentaneamente resolvendo a falta de médicos da atenção básica, que, quando forem embora, ficaremos sem novamente. (Usuário 10).

Acho que foi muito bom para nossa saúde, estão sendo um reforço nos atendimentos que o país estava precisando. (Usuário 8).

Com as falas acima percebemos que o Brasil precisa avançar no entendimento do processo de saúde e doença. A população brasileira não percebe a importância do cuidado ampliado, ou seja, o atendimento buscando a interação entre corpo, mente, social e espiritual.

O Programa Mais Médicos atinge os desejos e necessidades destes usuários, conforme os relatos analisados. Desse modo, demonstra, no caso deste município, ser um programa importante para a rede primária de saúde, proporcionando maior resolubilidade das demandas pelas equipes da ESF e satisfação da população.

\section{Conclusão}

O estudo objetivou identificar a implantação do Programa Mais Médicos em um município de pequeno porte, situado no Centro-Oeste paulista, na perspectiva dos funcionários e dos pacientes usuários dos serviços de ESF.

Sabe-se que a saúde pública vem passando por várias transformações ao longo do tempo. Dentre estas transformações, é possível perceber que os municípios estão gerenciando a saúde pública através da implantação da Estratégia de Saúde da Família (ESF). Com isso, é percebido um atendimento acessível projetado nas expressões das questões sociais presentes na comunidade onde a ESF é instalada, possibilitando que os funcionários tenham um conhecimento das dificuldades encontradas no cotidiano dos pacientes, bem como no estado de saúde e doença.

Por meio da análise dos dados desta pesquisa pode-se constatar que o Programa Mais Médicos do governo federal teve grande aceitação pela equipe de funcionários e pela população usuária das unidades de Saúde da Família avaliadas. Aceitação que foi confirmada nos discursos dos sujeitos entrevistados, mediante a referência a um bom relacionamento estabelecido entre todos, bem como o desempenho satisfatório no desenvolvimento do trabalho por parte dos médicos do programa.

Como se sabe, a ESF é instalada na maioria das vezes em áreas de maior vulnerabilidade social, onde a pobreza se torna presente. Muitas 


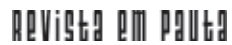

\} PROGRAMA MAIS MÉDICOS - CARRAPATO, J. F. L.; SILVA, R. V. C.; OTONDARO, E. C.; PLACIDELI, N.\} DOI: $10.12957 /$ REP.2016.27863

vezes é possível encontrar indivíduos com vínculo familiar rompido por uso indevido de substâncias psicoativas, violência, analfabetismo ou até mesmo por exclusão social.

Com isso, torna-se primordial que os profissionais das equipes de atenção primária da área da saúde trabalhem de forma consciente na realidade de cada família, sendo capazes de proporcionar um atendimento acolhedor de qualidade para conseguir orientar corretamente os pacientes.

A humanização e o acolhimento são fatores fundamentais para que haja um bom atendimento aos pacientes, fazendo com que eles se sintam seguros e sejam capazes de expor queixas e dúvidas sobre a doença que tenham ou que porventura venham a ter no decorrer dos anos.

Os pacientes mostraram-se bastante seguros e à vontade para serem assistidos nas consultas médicas com os profissionais do Programa Mais Médicos. Isto porque há realização de um atendimento humanizado, de forma ativa, no intuito de desvendar os reais sintomas presentes em cada paciente. Dessa maneira, os médicos desse programa prestam muitas orientações sobre os exames a serem feitos e as medicações a serem tomadas.

Nota-se também que há o atendimento médico domiciliar para os pacientes que não têm condições de se deslocar até as unidades de Saúde da Família para passar por consulta rotineira. Através desta ação os pacientes se sentem acolhidos e protegidos, proporcionando uma melhor interação e fortalecimento do vínculo entre paciente e médico.

Por fim, nota-se que a implantação deste programa foi de grande importância para a população do município investigado, pois os usuários, que muitas vezes tinham um atendimento médico rápido e objetivo, já que havia uma grande demanda de pacientes a serem atendidos em curto prazo, agora possuem um atendimento com mais tempo, mais tranquilidade e mais elaborado. Dessa forma, os profissionais médicos são capazes de examinar, conversar, fazer pedidos de exames, dar orientações e fazer prescrições de medicamentos.

O Programa Mais Médicos instituído no Brasil teve diversas repercussões em amplitude nacional, de caráter polêmico, com críticas fundamentadas por variados motivos. Entre estes, destacam-se as relacionadas à crise política e econômica que atualmente o país enfrenta; no entanto, o presente estudo demonstrou que os profissionais de equipes da ESF e, principalmente, os usuários destes serviços reconhecem no programa aspectos positivos. Desta forma, considera-se que o programa tem possibilitado o alcance dos princípios doutrinários do SUS, ou seja, a universalidade, a integralidade e a equidade, bem como os princípios organizacionais.

É importante que novas pesquisas sejam realizadas acerca do Programa Mais Médicos, sendo de cunho avaliativo, quantitativo e qualitativo, a fim de obter subsídios para uma avaliação fidedigna do programa. 


\section{Referências}

BRASIL. Constituição da República Federativa do Brasil. Brasília: Senado, 1988.

Lei n. 12.871 de 22 de outubro de 2013. Institui o Programa Mais Médicos, altera as Leis n. 8.745, de 9 de dezembro de 1993, e n. 6.932, de 7 de julho de 1981, e dá outras providências. 2013. Disponível em: <file:/ //C:/Us<sers/Usuario/Downloads/635-2484-1-PB.pdffile://C:/Users/Usua rio/Downloads/635-2484-1-PB.pdf.>. Acesso em: 12 de dez. 2016.

CAMPOS, G. W. de S. C. Mais Médicos e a construção de uma política de pessoal para a Atenção Básica no Sistema Único de Saúde (SUS). Revista Interface, 2015.

CARRYL, J.A. M.; ROSSATO, L.; PRADOS, R. M. N. Programa Mais Médicos. Rev. Diálogos Interdisciplinares, v. 5, n. 2, 2016.

IBGE, Censo demográfico 2010. Características da população e dos domicílios: resultados do universo. Rio de Janeiro: IBGE, 2011.

GIL, A. C. Como elaborar projetos de pesquisa. São Paulo: Editora Atlas, 2008.

ONOCKO-CAMPOS, R. T. et al. Avaliação de estratégias inovadoras na organização da atenção primária à saúde. Rev. Saúde Pública, n. 46, 2012.

RIBEIRO, R. C. Programa Mais Médicos - um equívoco conceitual. Ciência \& Saúde Coletiva, 2015.

RICHARDSON, J. R. Pesquisa social: métodos e técnicas. São Paulo: Atlas, 1985.

SOUSA, M. F. de. O programa saúde da família no Brasil: análise do acesso à atenção básica. Rev. Bras. Enferm., v. 61, n. 2, mar./abr. 2008.

STARFIELD, B. Atenção primária: equilíbrio entre as necessidades de saúde, serviços e tecnologia. Brasília: Unesco; Ministério da Saúde, 2002.

VASCONCELOS, M.; GRILLO, M. J. C; SOARES, S. M. Práticas educativas em atenção básica à saúde. Tecnologias para abordagem ao indivíduo, família e comunidade. Belo Horizonte: Editora UFMG; NESCON, 2009.

Recebido em 26 de maio de 2016.

Aprovado para publicação em 17 de novembro de 2016.

DOI: 10.12957/rep.2016.27863

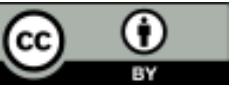

A Revista Em Pauta: Teoria Social e Realidade Contemporânea está licenciada com uma Licença Creative Commons Atribuição 4.0 Internacional. 